\title{
Age determinations of metal-poor field stars
}

\author{
A. Weiss ${ }^{1}$, M. Salaris ${ }^{1,2}$ and D. Rohr ${ }^{1}$ \\ ${ }^{1}$ Max-Planck-Institut für Astrophysik, Karl-Schwarzschild-Str. 1, 85276 Garching, Germany \\ email: aweiss@mpa-garching.mpg.de \\ ${ }^{2}$ Astrophysics Research Institute, Liverpool John Moores University, Twelve Quays House, \\ Egerton Wharf, Birkenhead, CH41 1LD, UK; email: ms@astro.livjm.ac.uk
}

\begin{abstract}
To determine ages of individual (field) stars, composition, mass and distance should be known accurately, which is usually not the case. An alternative way is to use turn-off colours of field star populations. This method led in the past to ages significantly higher than those of globular clusters of the same metallicity. We show that colour-based relative ages between the field and cluster population indicate that they have similar if not equal ages. First steps using large samples of stars from SDSS are presented.
\end{abstract}

Keywords. Stars: Population II, stars: subdwarfs, stars: evolution

\section{Individual stars}

To establish the evolution, in particular the chemical evolution of the Milky Way, we need accurate ages of the various subsystems. While there is now a widespread agreement about the ages of Globular Clusters, the same cannot be said for field stars. If the star's mass, distance and composition is known, it can be put on an evolutionary track and its luminosity yields its age. This we have done in Weiss \& Schlattl (2000) for stars analysed by Fuhrmann (1998), which probably have the best stellar parameter determinations available. Nevertheless, the estimated mass uncertainty of $5 \%$ results in determined ages of on average $11 \pm 4$ Gyr for such low-mass stars.

\section{Turn-off colours}

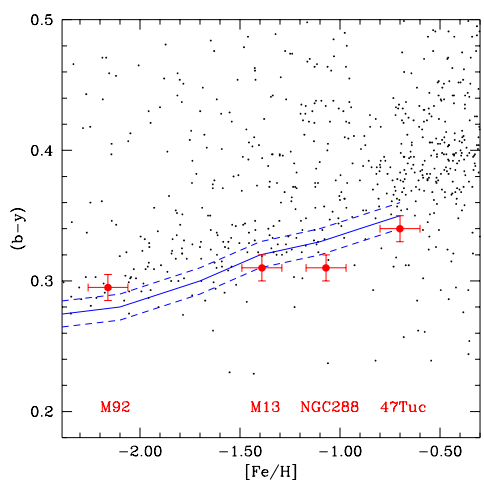

Without distance information, one can still try to identify the turn-off by looking for the bluest colour of a sample of field stars as function of metallicity and compare this to theoretical predictions. In Salaris \& Weiss (2001) we showed this for a sample of stars from Schuster \& Nissen (1989). Standard isochrones yielded ages of 16-18 Gyrs,
Figure 1. Strömgren TO-colours of 4 representative GCs (Grundahl, priv. comm.) compared to the sample of 411 field stars by Schuster et al. (2004). The (blue) lines denote the drop in stellar density interpreted as the TO and its uncertainty. The clusters have ages of 11 to 12 Gyr (from 47 Tuc to M92). 
while calculations including gravitational settling result in more acceptable ages of 1112 Gyr. The reason is that this method depends solely on the TO-colours, and the latter isochrones are redder. In Figure 1 we show an empirical comparison between the (Strömgren-)colours of the recent subdwarf sample by Schuster et al. (2004) with those of four globular clusters (Grundahl 2004, private communication), for which Salaris \& Weiss (2002) determined ages of 11 to 12 Gyr. Obviously, the direct comparison yields approximately the same ages for both halo components.
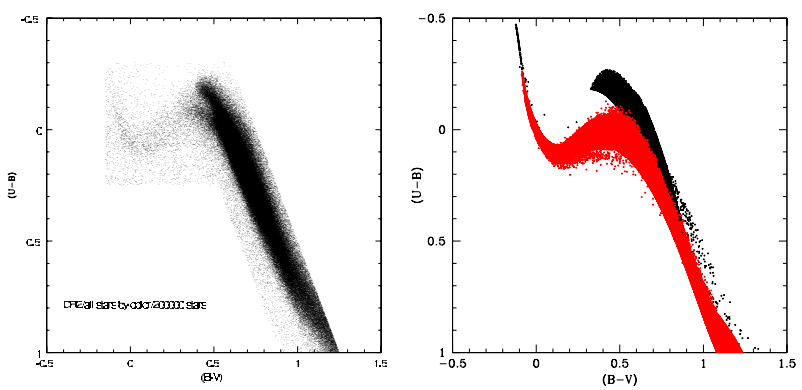

Figure 2. SDSS stars (left) from DR2 shown in an (U-B) $-(\mathrm{B}-\mathrm{V})$ diagram and selected by $r$-magnitude $<17$. Right: simulation; red: solar neighbourhood; black: halo stars with Gaussian distribution around $[\mathrm{Fe} / \mathrm{H}]=-1.6$ and ages of $12-13$ Gyr.

The future: large samples. The Sloan Digital Sky Survey (SDSS) is already providing several hundred thousands of stars with accurate photometric data. In addition, of order ten thousand stars have medium-resolution spectra, from which metallicities can be estimated quite reliably. Figure 2 (left) shows a colour-colour-diagram of SDSS/DR2 stellar objects brighter than 17 th magnitude in the $r$-band. To the right we show a simulation of the same diagram with a solar neighbourhood and a metal-poor population. The TO for the latter is clearly identifiable. These are the stars metallicities would be needed for. To this end we used the method by Beers et al. 1990 to estimate metallicities from the Ca HK lines. While this is not yet completed, Figure 3 shows a preliminary result: stars with $[\mathrm{Fe} / \mathrm{H}]$ as determined by us in the TO-region of the colour-colour-diagram. Our method is very fast and reliable at an average level of about $0.3 \operatorname{dex}$ in $[\mathrm{Fe} / \mathrm{H}]$.

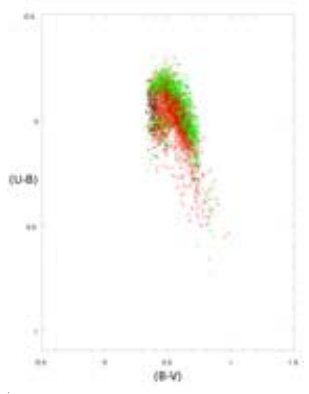

\section{References}

Beers, T.C., Preston, G.W., Shectman, S.A, \& Kage J.A., 1990, AJ 100, 849

Fuhrmann, K., 1998, A\&A 388, 161

Salaris, M. \& Weiss, A., 2001, in: von Hippel, T., et al. (eds.), Astrophysical Ages and Timescales, ASP Conf. Ser. (Astron. Soc. Pacific, San Francisco), vol. 245, p. 367

Salaris, M. \& Weiss, A., 2002, A $\& A$ 388, 492

Schuster, W.J. \& Nissen, P.E., 1989, A\&A 221, 65

Schuster, W.J., Beers, T.C., Michel, R., et al. , 2004, A\&A 422, 527

Weiss, A. \& Schlattl, H., 2000, A\&A Suppl. 144, 487 